\title{
Weighted norm inequalities for vector-valued singular integrals on homogeneous spaces
}

\author{
by \\ Sergio Antonio Tozoni (Campinas)
}

\begin{abstract}
Let $X$ be a homogeneous space and let $E$ be a UMD Banach space with a normalized unconditional basis $\left(e_{j}\right)_{j \geq 1}$. Given an operator $T$ from $L_{\mathrm{c}}^{\infty}(X)$ to $L^{1}(X)$, we consider the vector-valued extension $\widetilde{T}$ of $T$ given by $\widetilde{T}\left(\sum_{j} f_{j} e_{j}\right)=\sum_{j} T\left(f_{j}\right) e_{j}$. We prove a weighted integral inequality for the vector-valued extension of the Hardy-Littlewood maximal operator and a weighted Fefferman-Stein inequality between the vector-valued extensions of the Hardy-Littlewood and the sharp maximal operators, in the context of Orlicz spaces. We give sufficient conditions on the kernel of a singular integral operator to have the boundedness of the vector-valued extension of this operator on $L^{p}(X, W d \mu ; E)$ for $1<p<\infty$ and for a weight $W$ in the Muckenhoupt class $A_{p}(X)$. Applications to singular integral operators on the unit sphere $S^{n}$ and on a finite product of local fields $\mathbb{K}^{n}$ are given. The versions of all these results for vector-valued extensions of operators on functions defined on a homogeneous space $X$ and with values in a UMD Banach lattice are also given.
\end{abstract}

1. Introduction. The UMD property for Banach spaces plays a central role in the development of vector-valued Fourier analysis. Although they have been extensively studied (see e.g. $[5,3,4,21,20,11]$ ), we point out that all the maximal operators and singular integral operators considered in these studies act on functions defined on the Euclidian space $\mathbb{R}^{n}$ or on the torus $T^{n}$.

J. Bourgain [3] extended a result for vector-valued singular integral operators due to Benedek, Calderón and Panzone [1] to the context of UMD Banach spaces. The main goal of this paper is to prove a weighted extension of the result of J. Bourgain for vector-valued singular integral operators of functions defined on a homogeneous space (Theorem 1.4).

Let $E$ be a Banach space with a normalized unconditional basis $\left(e_{j}\right)_{j \geq 1}$ and let $S$ be the square function operator defined on martingales. Bourgain proved in [3] that the vector-valued extension $\widetilde{S}$ of $S, \widetilde{S}\left(\sum_{j} f_{j} e_{j}\right)=$

2000 Mathematics Subject Classification: Primary 42B20, 42B25; Secondary 60G46.

Key words and phrases: singular integral, maximal function, homogeneous space, UMD Banach space, $A_{p}$-weights. 
$\sum_{j} S\left(f_{j}\right) e_{j}$, is bounded on $L^{p}([0,1], d t ; E)$ if and only if $E$ has the UMD property. In our paper, the necessity of the UMD condition is stated as Theorem 2.2 and it is a consequence of the above-mentioned result by Bourgain.

The main difficulty compared to the Euclidian situation is in the transference method given in Section 3, which allows us to get an integral estimate between continuous maximal operators from harmonic analysis, using an integral estimate between dyadic maximal operators from martingale theory. This method for the Euclidean space was introduced by C. Fefferman and E. M. Stein in [8], when they proved the boundedness of a vector-valued extension of the Hardy-Littlewood maximal operator on $L^{p}\left(\mathbb{R}^{n}, d x ; \ell^{q}\right)$, using the boundedness of a dyadic maximal operator on $L^{p}\left([0,1]^{n}, d x ; \ell^{q}\right)$.

Several important singular integral operators on homogeneous spaces were studied, e.g., in Coifman-Weiss [7], Korányi-Vági [16], Levine [17] and Phillips-Taibleson [19]. We show that Theorem 1.4 can be applied to study vector-valued extensions of several singular integral operators from these works.

In Section 2 we study weighted integral estimates for vector-valued extensions of maximal operators from martingale theory in the context of Orlicz spaces.

In Section 3 we apply the results of Section 2 to prove a weighted integral inequality for a vector-valued extension of the Hardy-Littlewood maximal operator (Theorem 1.1) and a weighted Fefferman-Stein inequality between vector-valued extensions of the Hardy-Littlewood and the sharp maximal operators (Theorem 1.2), in the context of Orlicz spaces.

In Section 4 we study singular integral operators. The proofs of Theorems 1.3, 1.4 and Corollary 1.1 are in Section 4.

In the present section we give the statements of the main results of this paper.

Corollaries 1.1 and 1.2 are applications to vector-valued singular integral operators on functions defined on the unit sphere $S^{n}$ and on a finite product of local fields $\mathbb{K}^{n}$, respectively.

In Theorems 1.5-1.7 we consider vector-valued extensions of operators for functions defined on a homogeneous space $X$ and with values in a UMD Banach lattice.

Let $G$ be a locally compact Hausdorff topological group with unit element $e, H$ a compact subgroup of $G$, and $\pi: G \rightarrow G / H$ the canonical map. Let $d g$ denote a left Haar measure on $G$, which we assume to be normalized in the case of $G$ compact. If $A$ is a Borel subset of $G$, we denote by $|A|$ the Haar measure of $A$. The homogeneous space $X=G / H$ is the set of all left cosets $\pi(g)=g H, g \in G$, provided with the quotient topology. The Haar 
measure $d g$ induces a measure $\mu$ on the Borel $\sigma$-field on $X$. For $f \in L^{1}(X)$,

$$
\int_{X} f(x) d \mu(x)=\int_{G} f \circ \pi(g) d g .
$$

The measure $\mu$ on $X$ is invariant under the action of $G$, that is, if $f \in L^{1}(X)$, $g \in G$ and $R_{g} f(x)=f\left(g^{-1} x\right)$, then

$$
\int_{X} f(x) d \mu(x)=\int_{X} R_{g} f(x) d \mu(x) .
$$

A quasi-distance on $X$ is a map $d: X \times X \rightarrow[0, \infty)$ satisfying:

(i) $d(x, y)=0$ if and only if $x=y$;

(ii) $d(x, y)=d(y, x)$ for all $x, y \in X$;

(iii) $d(g x, g y)=d(x, y)$ for all $g \in G, x, y \in X$;

(iv) there exists a constant $\eta \geq 1$ such that for all $x, y, z \in X$,

$$
d(x, y) \leq \eta[d(x, z)+d(z, y)]
$$

(v) the balls $B(x, l)=\{y \in X: d(x, y)<l\}, x \in X, l>0$, are relatively compact and measurable, and the balls $B(\mathbb{1}, l), l>0$, form a basis of neighborhoods of $\mathbb{1}=\pi(e)$;

(vi) (doubling condition) there exists a constant $A \geq 1$ such that for all $l>0$ and $x \in X$,

$$
\mu(B(x, 2 l)) \leq A \mu(B(x, l)) .
$$

Given a quasi-distance $d$ on $X$, there exists a distance $\varrho$ on $X$ and a positive real number $\gamma$ such that $d$ is equivalent to $\varrho^{\gamma}$ (see [18]). Therefore the family of $d$-balls is equivalent to the family of $\varrho^{\gamma}$-balls, and $\varrho^{\gamma}$-balls are open sets. We can show that $\mu(B(x, l))>0$ for $x \in X, l>0$, and that $X$ is separable.

In this paper $X$ will denote a homogeneous space provided with a quasidistance $d$.

Given a Banach space $E$ with norm $\|\cdot\|$ and a positive locally integrable function $W$ on $X$, we denote by $L^{p}(X, W d \mu ; E)$ or $L_{E}^{p}(W), 1 \leq p<\infty$, the Bochner-Lebesgue space consisting of all $E$-valued (strongly) measurable functions $f$ defined on $X$ such that

$$
\|f\|_{L_{E}^{p}(W)}=\left(\int_{X}\|f(x)\|^{p} W(x) d \mu(x)\right)^{1 / p}<\infty .
$$

We write $L_{E}^{p}(W)=L^{p}(W)$ when $E=\mathbb{R}$, and $L_{E}^{p}(W)=L_{E}^{p}(X)=L_{E}^{p}$ when $W=1$. For the definition of the UMD property of a Banach space see e.g. $[5,3,4,21]$.

We say that a non-decreasing real-valued continuous function $\Phi$ on $[0, \infty)$ with $\Phi(0)=0$ satisfies the $\triangle_{2}$-condition if there exists a constant $c>0$ such that 


$$
\Phi(2 \lambda) \leq c \Phi(\lambda), \quad \lambda>0 .
$$

We put $\Phi(\infty)=\lim _{\lambda \rightarrow \infty} \Phi(\lambda)$.

Let $W$ be a positive locally integrable function on $X$ and let $1<p<\infty$. If there exists a constant $C$ such that

$$
\left(\frac{1}{\mu(B)} \int_{B} W d \mu\right)\left(\frac{1}{\mu(B)} \int_{B} W^{-1 /(p-1)} d \mu\right)^{p-1} \leq C
$$

for all balls $B=B(x, l), l>0, x \in X$, we say that $W$ is a weight in the Muckenhoupt class $A_{p}(X)$. If $W \in A_{p}(X)$, we denote by $C(p, W)$ the smallest constant $C$ that satisfies (1.2). The class $A_{\infty}(X)$ is defined as the union of the classes $A_{p}(X)$ for $1<p<\infty$. In Remark 3.1 we prove that for $p_{1}, p_{2} \in X$ and $0<\gamma_{1}, \gamma_{2}<1$ the weight

$$
W(x)=\frac{\left(\mu\left(B\left(\mathbb{1}, d\left(p_{2}, x\right)\right)\right)\right)^{\gamma_{2}(p-1)}}{\left(\mu\left(B\left(\mathbb{1}, d\left(p_{1}, x\right)\right)\right)\right)^{\gamma_{1}}}
$$

is in the class $A_{p}(X)$.

Let $f$ be a real-valued locally integrable function on $X$. The HardyLittlewood maximal operator $M$ and the sharp maximal operator $M^{\sharp}$ are defined at $f$ by

$$
\begin{aligned}
M f(x) & =\sup _{B} \frac{1}{\mu(B)} \int_{B}|f(y)| d \mu(y), \\
M^{\sharp} f(x) & =\sup _{B} \frac{1}{\mu(B)} \int_{B}\left|f(y)-f_{B}\right| d \mu(y),
\end{aligned}
$$

where

$$
f_{B}=\frac{1}{\mu(B)} \int_{B} f(y) d \mu
$$

and the suprema are taken over all balls $B$ such that $x \in B$.

The following theorem extends results for the Hardy-Littlewood maximal operator given in $[8,3,26]$.

THEOREM 1.1. Let $E$ be a Banach space with the UMD property and with a normalized unconditional basis $\left(e_{j}\right)_{j \geq 1}$, let $\Phi$ be a non-decreasing convex function on $[0, \infty)$ with $\Phi(0)=0$ and satisfying the $\triangle_{2}$-condition, and let $W \in A_{\infty}(X)$. Then there exists a constant $C$, depending only on $E, \Phi, X$ and $W$, such that

$$
\int_{X} \Phi\left(\sup _{k \geq 1}\left\|\sum_{j=1}^{k} M f_{j} e_{j}\right\|\right) W d \mu \leq C \int_{X} \Phi(M(\|f\|)) W d \mu
$$

for all $f=\sum_{j} f_{j} e_{j} \in L_{E}^{1}$. Moreover, if $1<p<\infty, W \in A_{p}(X)$ and $f \in L_{E}^{p}(W)$, then $\sum_{j} M f_{j} e_{j}$ converges in $L_{E}^{p}(W)$ to a function $\widetilde{M} f$ and the operator $\widetilde{M}$ is bounded on $L_{E}^{p}(W)$. 
There is an intimate relation between the Hardy-Littlewood maximal operator and the sharp maximal operator. This relation is contained in the inequality $\|M f\|_{p} \leq C\left\|M^{\sharp} f\right\|_{p}, f \in L^{p_{0}}\left(\mathbb{R}^{n}\right), 0<p_{0} \leq p<\infty$. This inequality is known as the Fefferman-Stein inequality and it was proved in [9]. A weighted extension of this inequality and an unweighted extension for functions defined on a space of homogeneous type (in particular on a homogeneous space) are well known. The following theorem gives a weighted vector-valued extension of the Fefferman-Stein inequality for functions defined on a homogeneous space $X$.

TheOREM 1.2. Let $E$ be a Banach space with the UMD property and with a normalized unconditional basis $\left(e_{j}\right)_{j \geq 1}$, let $\Phi$ be a non-decreasing convex function on $[0, \infty)$ with $\Phi(0)=0$ and satisfying the $\triangle_{2}$-condition, and let $W \in A_{\infty}(X)$. Then there exists a constant $C$, depending only on $E$, $\Phi, X$ and $W$, such that for all $f=\sum_{j} f_{j} e_{j} \in \bigcup_{p>1} L_{E}^{p}$,

$$
\int_{X} \Phi\left(\left\|\sum_{j=1}^{\infty} M f_{j} e_{j}\right\|\right) W d \mu \leq C \int_{X} \Phi\left(\left\|\sum_{j=1}^{\infty} M^{\sharp} f_{j} e_{j}\right\|\right) W d \mu .
$$

We say that a linear operator $T$ defined on $L_{c}^{\infty}(X)$ and with values in the space of all measurable functions, is a singular integral operator if the following conditions hold:

(i) $T$ has a bounded extension on $L^{r}(X)$ for some $r, 1<r \leq \infty$;

(ii) there exists a kernel $K \in L_{\text {loc }}^{1}(X \times X \backslash \triangle), \triangle=\{(x, x): x \in X\}$, such that

$$
T f(x)=\int_{X} K(x, y) f(y) d \mu(y)
$$

for all $f \in L_{\mathrm{c}}^{\infty}(X)$ and almost all $x \notin \operatorname{supp} f$.

Let $T$ be a singular integral operator with a kernel $K$. We say that $K$ satisfies the condition $\left(H_{\infty}\right)$ if

$$
|K(x, y)-K(x, \mathbb{1})| \leq C \frac{d(y, \mathbb{1})}{d(x, \mathbb{1}) \mu(B(\mathbb{1}, d(x, \mathbb{1}))}
$$

whenever $d(x, \mathbb{1})>2 d(y, \mathbb{1}), \mathbb{1}=\pi(e)$. If $K^{\prime}(x, y)=K(y, x)$ satisfies $\left(H_{\infty}\right)$ we say that $K$ satisfies $\left(H_{\infty}^{\prime}\right)$.

The following theorem is proved in Section 4.

THEOREM 1.3. Let $E$ be a Banach space with the UMD property and with a normalized unconditional basis $\left(e_{j}\right)_{j \geq 1}$. Let $1<p<\infty, W \in A_{p}(X)$, and let $\left(T_{j}\right)_{j \geq 1}$ be a sequence of operators from $L^{p}(W)$ to $L^{p}(W)$ such that for every $r>1$ there exists a constant $C_{r}$ such that

$$
M^{\sharp}\left(T_{j} f\right)(x) \leq C_{r} M_{r} f(x), \quad f \in L_{\mathrm{c}}^{\infty}(X), j \geq 1 .
$$


Then for all $f=\sum_{j} f_{j} e_{j} \in L_{E}^{p}(W)$ the series $\sum_{j} T_{j} f_{j} e_{j}$ converges in $L_{E}^{p}(W)$ and there exists a positive constant $C_{p}$ such that

$$
\left\|\sum_{j=1}^{\infty} T_{j} f_{j} e_{j}\right\|_{L_{E}^{p}(W)} \leq C_{p}\left\|\sum_{j=1}^{\infty} f_{j} e_{j}\right\|_{L_{E}^{p}(W)} .
$$

It is easy to see that the condition $\left(H_{\infty}\right)$ for the kernel $K$ of a singular integral operator implies Hörmander's condition $\left(H_{1}\right)$ :

$$
\int_{d(x, \mathbb{1})>2 d(y, \mathbb{1})}|K(x, y)-K(x, \mathbb{1})| d \mu(x) \leq C<\infty .
$$

Hörmander's condition was studied by R. R. Coifman and G. Weiss [7], A. Korányi and S. Vági [16] and B. Bordin and D. L. Fernandez [2]. It was proved that if the kernel $K$ satisfies $\left(H_{1}\right)$ and $\left(H_{1}^{\prime}\right)$, then the singular integral operator is bounded on $L^{p}(X)$ for $1<p<\infty$. The next result follows immediately from Lemma 4.2 in Section 4 and Theorem 1.3.

THEOREM 1.4. Let $E$ be a Banach space with the UMD property and with a normalized unconditional basis $\left(e_{j}\right)_{j \geq 1}$. Let $1<p<\infty, W \in A_{p}(X)$, and let $T$ be a singular integral operator. Assume that the kernel $K$ of $T$ satisfies $\left(H_{\infty}\right),\left(H_{\infty}^{\prime}\right)$ and $K(g x, g y)=K(x, y)$ for all $x, y \in X, g \in G$. Then for all $f=\sum_{j} f_{j} e_{j} \in L_{E}^{p}(W)$ the series $\sum_{j} T f_{j} e_{j}$ converges in $L_{E}^{p}(W)$ and there exists a positive constant $C_{p}$ such that

$$
\left\|\sum_{j=1}^{\infty} T f_{j} e_{j}\right\|_{L_{E}^{p}(W)} \leq C_{p}\left\|\sum_{j=1}^{\infty} f_{j} e_{j}\right\|_{L_{E}^{p}(W)} .
$$

Theorem 1.4 for the Euclidian space $\mathbb{R}^{n}$ and $W=1$ was proved by Bourgain [3]; it was also studied in [21]. For $W=1$ and $E=\ell^{q}, 1<q<\infty$, but for more general spaces $X$ (of homogeneous type) it was proved in $[2,22]$. Theorem 1.3 for $X=\mathbb{R}^{n}$ and $W=1$ was proved in [21].

Let us consider the unit sphere $S^{n}=\left\{x \in \mathbb{R}^{n+1}:|x|=1\right\}$ provided with the Lebesgue measure $d \sigma$ and with the Euclidian distance $d(x, y)=|x-y|$ and let $\mathbb{1}=(1,0, \ldots, 0)$. Given $p_{1}, p_{2} \in S^{n}$ and $0<\gamma_{1}, \gamma_{2}<1$, the weight $W(x)=\left|p_{2}-x\right|^{n \gamma_{2}(p-1)}\left|p_{1}-x\right|^{-n \gamma_{1}}$ is in the class $A_{p}\left(S^{n}\right)$.

A kernel $K \in L_{\text {loc }}^{1}\left(S^{n} \times S^{n} \backslash \triangle\right)$ satisfies the condition $\left(H_{\infty}\right)$ if there exists a constant $C$ such that for $x, y \in S^{n}$ with $|x-\mathbb{1}|>2|y-\mathbb{1}|$ we have

$$
|K(x, y)-K(x, \mathbb{1})| \leq C \frac{|y-\mathbb{1}|}{|x-\mathbb{1}|^{n+1}} .
$$

For $0 \leq r \leq 1, i, j \in\{1, \ldots, n+1\}$ and $x, y \in S^{n}(x \neq y$ for $r=1)$, we 
define the kernels $s_{r}, t_{r}, K_{i, j}^{r}$ and $K$ by

$$
\begin{aligned}
s_{r}(x, y) & =\frac{2}{\omega_{n}} \frac{y-(y \cdot x) x}{|y-r x|^{n+1}}, & K_{i, j}^{r}(x, y) & =\frac{x_{i} y_{j}-x_{j} y_{i}}{|y-r x|^{n+1}}, \\
t_{r}(x, y) & =\frac{n-2}{2 r} \int_{0}^{r} s_{\varrho}(x, y) d \varrho, & K(x, y) & =-\int_{0}^{1} P_{r}(x, y) d r
\end{aligned}
$$

where $P_{r}(x, y)$ denotes the Poisson kernel

$$
P_{r}(x, y)=\frac{1}{\omega_{n}} \frac{1-r^{2}}{|y-r x|^{n+1}} .
$$

Let $q_{r}=s_{r}+t_{r}, 0 \leq r \leq 1$. For $f \in L^{\infty}\left(S^{n}\right)$ we define the operators $R_{r}$, $R_{i, j}^{r}, 0 \leq r \leq 1$ and $i, j \in\{1, \ldots, n+1\}$, and $\Lambda$ by

$$
\begin{aligned}
R_{r} f(x) & =\int_{S^{n}} q_{r}(x, y) f(y) d \sigma(y), \\
R_{i, j}^{r} f(x) & =\int_{S^{n}} K_{i, j}^{r}(x, y) f(y) d \sigma(y), \\
\Lambda f(x) & =\int_{S^{n}} K(x, y) f(y) d \sigma(y),
\end{aligned}
$$

with $x \in S^{n}$ if $0 \leq r<1$ and $x \notin \operatorname{supp} f$ if $r=1$.

The operator $R=R_{1}$ is called the Riesz transform on $S^{n}$ and it was proved in Korányi-Vági [16, p. 636] that: $\lim _{r \rightarrow 1} R_{r} f=R f$ exists a.e. and in $L^{p}\left(S^{n}\right), 1<p<\infty$; the operators $R_{r}$ are uniformly bounded on $L^{p}\left(S^{n}\right)$, and $q_{r}(g x, g y)=q_{r}(x, y)$ for all $x, y \in S^{n}, g \in S O(n+1)$. The operators $R_{i, j}^{r}$ were considered in Coifman-Weiss [7, p. 76]. They are uniformly bounded on $L^{2}\left(S^{n}\right)$ and $K_{i, j}^{r}(g x, g y)=K_{i, j}^{r}(x, y)$ for all $x, y \in S^{n}, g \in S O(n+1)$. The operator $\Lambda$ was studied in Levine [17, p. 508], where it was proved that: it is bounded on $L^{p}\left(S^{n}\right)$ for $1 \leq p \leq \infty$; if $Y_{k}$ is a spherical harmonic of degree $k$ then $\Lambda Y_{k}=-Y_{k} /(k+1)$, and $K(g x, g y)=K(x, y)$ for all $x, y \in S^{n}$, $g \in S O(n+1)$.

In Section 4 we prove the following result.

Corollary 1.1. Let $E$ be a Banach space with the UMD property and with a normalized unconditional basis $\left(e_{j}\right)_{j \geq 1}$. Let $1<p<\infty, W \in A_{p}\left(S^{n}\right)$ and $T \in\left\{R_{r}, R_{i, j}^{r}, \Lambda: 0 \leq r \leq 1,1 \leq i, j \leq n+1\right\}$. Then there exists $a$ constant $C_{p}$ such that

$$
\left\|\sum_{j=1}^{\infty} T f_{j} e_{j}\right\|_{L_{E}^{p}(W)} \leq C_{p}\left\|\sum_{j=1}^{\infty} f_{j} e_{j}\right\|_{L_{E}^{p}(W)}
$$

for all $f=\sum_{j} f_{j} e_{j} \in L^{p}\left(S^{n}, W d \sigma ; E\right)$. 
A local field is any locally compact, non-discrete and totally disconnected field. Let $\mathbb{K}$ be a fixed local field and $d x$ be a Haar measure on the additive group $\mathbb{K}^{+}$of $\mathbb{K}$. The measure of a measurable set $A$ of $\mathbb{K}$ with respect to $d x$ is denoted by $|A|$. Let $m$ be the modular function for $\mathbb{K}^{+}$, that is, $m(\lambda)|A|=|\lambda A|$ for $\lambda \in \mathbb{K}$ and $A \subset \mathbb{K}$ measurable. We also write $|x|=m(x)$. The sets

$$
\mathbb{D}=\{x \in \mathbb{K}:|x| \leq 1\} \quad \text { and } \quad \mathbb{B}=\{x \in \mathbb{K}:|x|<1\}
$$

are the ring of integers of $\mathbb{K}$ and the unique maximal ideal of $\mathbb{D}$, respectively. Let $q=p^{c}$ ( $p$ prime) be the order of the finite field $\mathbb{D} / \mathbb{B}$ and let $\pi$ be a fixed element of $\mathbb{B}$ of maximum absolute value. The Haar measure $d x$ is normalized so that $|\mathbb{D}|=1$ and thus $|\pi|=|\mathbb{B}|=q^{-1}$.

A local field $\mathbb{K}$ has a natural sequence of partitions into balls satisfying conditions (i) and (ii) of Lemma 3.1 in Section 3, when we consider the distance $d(x, y)=|x-y|$. It follows that Theorems 1.1 and 1.2 hold without the hypothesis of $\Phi$ being a convex function. The extension of these results to a finite product of local fields is an immediate consequence of a theorem of M. H. Taibleson (see [24, pp. 548-549]). Given $p_{1}, p_{2} \in \mathbb{K}$ and $0<\gamma_{1}, \gamma_{2}<1$, the weight $W(x)=\left|p_{2}-x\right|^{n \gamma_{2}(p-1)}\left|p_{1}-x\right|^{-n \gamma_{1}}$ is in the class $A_{p}(\mathbb{K})$.

A kernel $K \in L_{\text {loc }}^{1}\left(\mathbb{K}^{n} \times \mathbb{K}^{n} \backslash \triangle\right)$ satisfies the condition $\left(H_{\infty}\right)$ if for $x, y \in \mathbb{K}^{n}$ with $|x|>|y|$ we have

$$
|K(x, y)-K(x, 0)| \leq C \frac{|y|}{|x|^{n+1}} .
$$

Let $\omega(x)$ be a function defined on $\mathbb{K}^{n}$ and satisfying:

$$
\begin{gathered}
\omega(x)=\omega\left(\pi^{j} x\right), \quad j \text { integer, } x \in \mathbb{K}^{n} \\
\int_{|x|=1} \omega(x) d x=0 ; \\
\left|\omega\left(x-\pi^{j} y\right)-\omega(x)\right| \leq C q^{-j}, \quad j \geq 1,|x|=|y|=1 .
\end{gathered}
$$

Then the kernel $\Psi(x, y)=\Psi(x-y)$, where

$$
\Psi(x)=\frac{\omega(x)}{|x|^{n}}, \quad x \in \mathbb{K}^{n} \backslash\{0\},
$$

satisfies $\left(H_{\infty}\right)$ and $\left(H_{\infty}^{\prime}\right)$. For $f \in L^{\infty}\left(\mathbb{K}^{n}\right)$ and $x \in \mathbb{K}^{n}, x \notin \operatorname{supp} f$, we define

$$
U f(x)=\int_{\mathbb{K}^{n}} \Psi(x-y) f(y) d y .
$$

The operator $U$ was studied in Phillips-Taibleson [19] and it was proved that $U$ is bounded on $L^{p}\left(\mathbb{K}^{n}\right)$ for $1<p<\infty$. Therefore the next corollary follows from Theorem 1.4. 
COROLlary 1.2. Let $E$ be a Banach space with the UMD property and with a normalized unconditional basis $\left(e_{j}\right)_{j \geq 1}$. Let $1<p<\infty$ and $W \in$ $A_{p}\left(\mathbb{K}^{n}\right)$. Then there exists a constant $C_{p}$ such that

$$
\left\|\sum_{j=1}^{\infty} U f_{j} e_{j}\right\|_{L_{E}^{p}(W)} \leq C_{p}\left\|\sum_{j=1}^{\infty} f_{j} e_{j}\right\|_{L_{E}^{p}(W)}
$$

for all $f=\sum_{j} f_{j} e_{j} \in L^{p}\left(\mathbb{K}^{n}, W d y ; E\right)$.

Now let $E$ be a Banach lattice of real-valued measurable functions on a $\sigma$-finite measure space $(Y, \mathcal{B}, \nu)$. The absolute value of $h \in E$ is given by $|h|(y)=|h(y)|, y \in Y$. We identify a function $f \in L_{E}^{p}(W)$ with a function defined on the product $X \times Y$ by setting $f(x)(y)=f(x, y)$. We denote by $L^{p}(W) \otimes E$ the set of all vector-valued functions $f$ of the type $f=\sum_{j=1}^{k} a_{j} f_{j}$ for $a_{j} \in E, f_{j} \in L^{p}(W)$ and for some integer $k \geq 1$. This set is a dense subspace of $L_{E}^{p}(W)$ for $1 \leq p<\infty$ and any weight $W$. Given an operator $T$ in $L^{p}(W)$, we define its extension $\bar{T}$ to $L^{p}(W) \otimes E$ (see Rubio de Francia [20]) by

$$
\bar{T} f(x, y)=T(f(\cdot, y))(x), \quad(x, y) \in X \times Y .
$$

A characterization of UMD Banach lattices in terms of the extension $\bar{M}$ of the Hardy-Littlewood maximal operator, when $X=\mathbb{R}^{n}$, was given by Bourgain [3] (see also [20]). Bourgain's characterization says that $E$ has the UMD property if and only if $\bar{M}$ is bounded on $L_{E}^{p}\left(\mathbb{R}^{n}\right)$ and on $L_{E^{\prime}}^{p^{\prime}}\left(\mathbb{R}^{n}\right)$ for some $p, 1<p<\infty$, where $p^{\prime}$ is the conjugate exponent of $p$ and $E^{\prime}$ is the dual space of $E$. The maximal operator $\bar{M}$ and other maximal operators of the same type were studied in $[11,14,12]$ for $X=\mathbb{R}^{n}$. In [11] new characterizations of UMD Banach lattices in terms of maximal operators are given.

In Section 2 we consider the maximal operators $N f=f_{\widetilde{N}}^{*}$ and $N^{\sharp} f=$ $f^{\sharp}$ from martingale theory and their vectorial extensions $\widetilde{N}\left(\sum_{j} f_{j} e_{j}\right)=$ $\sum_{j} f_{j}^{*} e_{j}, \widetilde{N}^{\sharp}\left(\sum_{j} f_{j} e_{j}\right)=\sum_{j} f_{j}^{\sharp} e_{j}$. The analogue of Theorem 2.4 for the operator $\bar{N}$ was proved in [26]. In the same way we can prove the analogue of Theorem 2.7 for the operators $\bar{N}$ and $\bar{N}^{\sharp}$. Proceeding as in Section 3, we can apply the inequalities obtained for $\bar{N}$ and $\bar{N}^{\sharp}$ to prove the following theorems.

THEOREM 1.5. Let E be a Banach lattice of real-valued measurable functions with the UMD property, let $\Phi$ be a non-decreasing convex function on $[0, \infty)$ with $\Phi(0)=0$ and satisfying the $\triangle_{2}$-condition, and let $W \in A_{\infty}(X)$. Then there exists a constant $C$, depending only on $E, \Phi, X$ and $W$, such that for all $f \in L^{1}(W) \otimes E$,

$$
\int_{X} \Phi(\|\bar{M} f\|) W d \mu \leq C \int_{X} \Phi(M(\|f\|)) W d \mu .
$$


THEOREM 1.6. Let $E$ be a Banach lattice of real-valued measurable functions with the $U M D$ property, let $\Phi$ be a non-decreasing convex function on $[0, \infty)$ with $\Phi(0)=0$ and satisfying the $\triangle_{2}$-condition, and let $W \in A_{\infty}(X)$. Then there exists a constant $C$, depending only on $E, \Phi, X$ and $W$, such that for all $f \in L^{1}(W) \otimes E$,

$$
\int_{X} \Phi(\|\bar{M} f\|) W d \mu \leq C \int_{X} \Phi\left(\left\|\bar{M}^{\sharp} f\right\|\right) W d \mu .
$$

If $\Phi(t)=t^{p}, 1<p<\infty$, then we can extend the operators $\bar{M}$ and $\bar{M}^{\sharp}$ by a limit process to all $L_{E}^{p}(W)$ and the above theorems will hold for these extensions. Proceeding as in Section 4 we can apply Theorems 1.5 and 1.6 to prove the following analogue of Theorem 1.4 for Banach lattices.

THEOREM 1.7. Let $E$ be a Banach lattice of real-valued measurable functions with the $U M D$ property, let $1<p<\infty, W \in A_{p}(X)$, and let $T$ be a singular integral operator. Assume that the kernel $K$ of $T$ satisfies $\left(H_{\infty}\right)$, $\left(H_{\infty}^{\prime}\right)$ and $K(g x, g y)=K(x, y)$ for all $x, y \in X, g \in G$. Then there exists a positive constant $C_{p}$ such that for all $f \in L^{p}(W) \otimes E$,

$$
\|\bar{T} f\|_{L_{E}^{p}(W)} \leq C_{p}\|f\|_{L_{E}^{p}(W)} .
$$

Moreover, the operator $\bar{T}$ can be continuously extended to all $L_{E}^{p}(W)$ and the above inequality holds for that extension and for all $f \in L_{E}^{p}(W)$.

Applying Theorem 1.7 we obtain the analogues of Corollaries 1.1 and 1.2 for the case of UMD Banach lattices.

2. Maximal operators in martingale theory. Let $(\Omega, \mathcal{F}, \mathbb{P})$ be a probability space and for each $k=0,1,2, \ldots$ let $\mathcal{A}_{k}$ be a partition of $\Omega$ into elements of $\mathcal{F}$ satisfying: $\mathbb{P}(Q)>0$ for all $Q \in \mathcal{A}_{k}$; the $\sigma$-field $\mathcal{F}$ is generated by the union $\mathcal{A}=\bigcup_{k=0}^{\infty} \mathcal{A}_{k}$; the partition $\mathcal{A}_{k+1}$ is a refinement of $\mathcal{A}_{k}$, that is, for each $Q \in \mathcal{A}_{k}$, there exists an integer $n_{Q} \geq 1$ and $Q_{1}, \ldots, Q_{n_{Q}} \in \mathcal{A}_{k+1}$ such that $Q=Q_{1} \cup \ldots \cup Q_{n_{Q}}$. We will denote by $\mathcal{F}_{k}$ the $\sigma$-field generated by $\mathcal{A}_{k}$ and we will always assume that the sequence $\left(\mathcal{A}_{k}\right)_{k \geq 0}$ is regular with respect to $\mathbb{P}$, that is, there exists an absolute constant $\theta \geq 1$ such that

$$
\mathbb{P}\left(Q_{1}\right) \leq \theta \mathbb{P}\left(Q_{2}\right)
$$

for all $Q_{1} \in \mathcal{A}_{k}$ and $Q_{2} \in \mathcal{A}_{k+1}$ with $Q_{2} \subset Q_{1}, k \geq 0$.

Given an $E$-valued integrable function $f: \Omega \rightarrow E$ we will also denote by $f$ the martingale $\left(f_{k}\right)_{k \geq 0}$, where $f_{k}=E\left[f \mid \mathcal{F}_{k}\right]$ is the conditional expectation of the function $f$ with respect to the $\sigma$-field $\mathcal{F}_{k}$. A stopping time is a function $T: \Omega \rightarrow\{0,1, \ldots, \infty\}$ such that $\{T \leq k\} \in \mathcal{F}_{k}$ for all $k \geq 0$. For a stopping time $T$ we denote by $\mathcal{F}_{T}$ the $\sigma$-field of all sets $A \in \mathcal{F}$ such that $A \cap\{T \leq k\}$ $\in \mathcal{F}_{k}$ for all $k \geq 0$. The martingale transform " $f$ stopped at $T$ " is defined by $f^{T}=\left(f_{k}^{T}\right)_{k \geq 0}, f_{k}^{T}(\omega)=f_{T(\omega) \wedge k}(\omega)$, and we write $f_{T}(\omega)=f_{T(\omega)}(\omega)$. We 
can show that

$$
E\left[I(A)\left\{f-f_{T}\right\} \mid \mathcal{F}_{k}\right]=I(A)\left(f_{k}-f_{k}^{T}\right)
$$

for all integrable functions $f: \Omega \rightarrow E$, all stopping times $T$, all $k \geq 0$ and all $A \in \mathcal{F}_{T}$, where $I(A)$ is the indicator function of the set $A$.

For a real-valued integrable function $f$ we define the maximal functions

$$
\begin{aligned}
& f^{*}(x)=\sup _{k \geq 0}\left|f_{k}(x)\right|=\sup _{\substack{x \in Q \\
Q \in \mathcal{A}}} \frac{1}{\mathbb{P}(Q)}\left|\int_{Q} f d \mathbb{P}\right|, \\
& f^{\sharp}(x)=\sup _{k \geq 0} E\left[\left|f-f_{k}\right| \mid \mathcal{F}_{k}\right](x)=\sup _{\substack{x \in Q \\
Q \in \mathcal{A}}} \frac{1}{\mathbb{P}(Q)} \int_{Q}\left|f-f_{Q}\right| d \mathbb{P},
\end{aligned}
$$

where

$$
f_{Q}=\frac{1}{\mathbb{P}(Q)} \int_{Q} f d \mathbb{P} .
$$

For an integer $n \geq 0$ we define $f_{n}^{*}=\left(f_{n}\right)^{*}, f_{n}^{\sharp}=\left(f_{n}\right)^{\sharp}$.

It is well known (see [10]) that

$$
\left\|f^{*}\right\|_{p} \leq C_{p}\left\|f^{\sharp}\right\|_{p}, \quad 1<p<\infty, f \in L^{p}(\Omega, \mathcal{F}, \mathbb{P}) .
$$

We can prove (2.3) using the method known as the Calderón-Zygmund decomposition (see [9, Theorem 5, p. 153]), replacing the dyadic cubes of $\mathbb{R}^{n}$ by the elements of $\mathcal{A}$.

Given a positive integrable function $W$ on $\Omega$, we denote by $L^{p}(\Omega, \mathcal{F}$, $W d \mathbb{P} ; E)$ or $L_{E}^{p}(W), 1 \leq p<\infty$, the Bochner-Lebesgue space consisting of all $E$-valued (strongly) measurable functions $f$ defined on $\Omega$ such that

$$
\|f\|_{L_{E}^{p}(W)}=\left(\int_{\Omega}\|f(\omega)\|^{p} W(\omega) d \mathbb{P}(\omega)\right)^{1 / p}<\infty .
$$

We write $L_{E}^{p}(W)=L^{p}(W)$ when $E=\mathbb{R}$, and $L_{E}^{p}(W)=L_{E}^{p}(\Omega)=L_{E}^{p}$ when $W=1$.

Let $W$ be a positive integrable function on $\Omega$ and let $1<p<\infty$. If there exists a constant $C$ such that

$$
\left(\frac{1}{\mathbb{P}(Q)} \int_{Q} W d \mathbb{P}\right)\left(\frac{1}{\mathbb{P}(Q)} \int_{Q} W^{-1 /(p-1)} d \mathbb{P}\right)^{(p-1)} \leq C
$$

for all $Q \in \mathcal{A}$, we say that $W$ is a weight in the class $A_{p}(\mathcal{A})$. The class $A_{\infty}(\mathcal{A})$ is defined as the union of $A_{p}(\mathcal{A})$ for $1<p<\infty$.

Let $U$ be an operator on $L_{E}^{1}$ which to each $f \in L_{E}^{1}$ associates a nonnegative process $\left(U_{k} f\right)_{k \geq 0}$ with $U_{0} f=0$ and $U_{k} f \mathcal{F}_{k}$-measurable, $k \geq 0$. For a stopping time $T$ we denote by $U_{T}^{*}$ the maximal operator defined by

$$
U_{T}^{*} f(\omega)=\sup _{k \leq T(\omega)} U_{k} f(\omega) .
$$

We write $U^{*} f=U_{\infty}^{*} f$. 
Theorem $2.1([25])$. Let $W \in A_{\infty}(\mathcal{A})$ and let $U$ and $V$ be two operators on $L_{E}^{1}$ as above. Suppose that

$$
U_{T \wedge S}^{*} f=U_{T}^{*} f^{S}, \quad V_{T \wedge S}^{*} f=V_{T}^{*} f^{S}
$$

for all stopping times $T$ and $S$ and all $f \in L_{E}^{1}$. If there exists a constant $C$ such that

$$
E\left[\left\{U_{k}^{*} f-U_{T \wedge k}^{*} f\right\}^{2} \mid \mathcal{F}_{T}\right] \leq C E\left[\left\{V_{k}^{*} f\right\}^{2}|| \mathcal{F}_{T}\right]
$$

for all $k \geq 1$, all stopping times $T$ and all $f \in L_{E}^{1}$, then there exists a constant $C$ such that

$$
\int_{\Omega} \Phi\left(U^{*} f\right) W d \mathbb{P} \leq C \int_{\Omega} \Phi\left(V^{*} f\right) W d \mathbb{P}
$$

for all $f \in L_{E}^{1}$. The constant $C$ depends only on $W, \theta, \Phi$ and $E$, where $\theta$ is the constant in (2.1).

TheOREm $2.2([26])$. Let $E$ be a Banach space with the UMD property and with a normalized unconditional basis $\left(e_{j}\right)_{j \geq 1}$. Let $U$ and $V$ be two operators which to each real-valued integrable function on $\Omega$ associate nonnegative $\mathcal{F}$-measurable functions. Suppose that for any $Z \in A_{\infty}(\mathcal{A})$ there exists a constant $C_{Z}$, depending only on $Z$, such that

$$
\int_{\Omega} U(h) Z d \mathbb{P} \leq C_{Z} \int_{\Omega} V(h) Z d \mathbb{P}
$$

for all $h \in \bigcup_{k=0}^{\infty} L^{1}\left(\Omega, \mathcal{F}_{k}, \mathbb{P}\right)$. Then for all $1<p<\infty$ there exists a constant $C_{p}$ such that

$$
\left\|\sum_{j=1}^{\infty} U f_{j} e_{j}\right\|_{L_{E}^{p}} \leq C_{p}\left\|\sum_{j=1}^{\infty} V f_{j} e_{j}\right\|_{L_{E}^{p}}
$$

for all $f=\sum_{j} f_{j} e_{j} \in \bigcup_{k=0}^{\infty} L^{p}\left(\Omega, \mathcal{F}_{k}, \mathbb{P} ; E\right)$.

THEOREM $2.3([15])$. Let $W$ be a positive integrable function and let $1<$ $p<\infty$. Then $W \in A_{p}(\mathcal{A})$ if and only if the operator $f \mapsto f^{*}$ is bounded on $L^{p}(W)$.

Theorem 2.4 ([26]). Let $E$ be a Banach space with the UMD property and with a normalized unconditional basis $\left(e_{j}\right)_{j \geq 1}$, let $\Phi$ be a non-decreasing continuous function on $[0, \infty)$ with $\Phi(0)=0$ and satisfying the $\triangle_{2}$-condition, and let $W \in A_{\infty}(\mathcal{A})$. Then there exists a constant $C$, depending only on $E$, $\Phi$ and $W$, such that for all $f=\sum_{j} f_{j} e_{j} \in L_{E}^{1}$,

$$
\int_{\Omega} \Phi\left(\sup _{k \geq 1}\left\|\sum_{j=1}^{k} f_{j}^{*} e_{j}\right\|\right) W d \mathbb{P} \leq C \int_{\Omega} \Phi\left(\|f\|^{*}\right) W d \mathbb{P} .
$$


LEmma 2.1. There exists an absolute constant $C$ such that for all stopping times $T$, all $f \in L^{1}(\Omega, \mathcal{F}, \mathbb{P})$ and all integers $n \geq 0$,

$$
E\left[\left\{\left(f-f^{T}\right)_{n}^{*}\right\}^{2} \mid \mathcal{F}_{T}\right] \leq C E\left[\left\{\left(f-f^{T}\right)_{n}^{\sharp}\right\}^{2} \mid \mathcal{F}_{T}\right] .
$$

Proof. Fix $T, f, n$ and $A \in \mathcal{F}_{T}$ and consider the martingale $g=\left(g_{k}\right)_{k \geq 0}$, $g_{k}=E\left[I(A)\left\{f_{n}-f_{T \wedge n}\right\} \mid \mathcal{F}_{k}\right]$. From (2.2) it follows that

$$
g_{k}=I(A)\left(f_{k}^{n}-f_{k}^{T \wedge n}\right)
$$

and hence

$$
g_{n}^{*}=I(A)\left(f-f^{T}\right)_{n}^{*}
$$

and

$$
\left|g_{n}-g_{k}\right|=I(A)\left|\left(f-f^{T}\right)_{n}-\left(f-f^{T}\right)_{k}\right|, \quad 1 \leq k \leq n .
$$

Since $A \cap\{T \leq k\} \in \mathcal{F}_{k}$ we have

$$
\begin{aligned}
E\left[\left|g_{n}-g_{k}\right| \mid \mathcal{F}_{k}\right]= & E\left[I(A \cap\{T \leq k\})\left|\left(f-f^{T}\right)_{n}-\left(f-f^{T}\right)_{k}\right| \mid \mathcal{F}_{k}\right] \\
& +E\left[I(A \cap\{T>k\})\left|\left(f-f^{T}\right)_{n}-\left(f-f^{T}\right)_{k}\right| \mid \mathcal{F}_{k}\right] \\
= & I(A) E\left[\left|\left(f-f^{T}\right)_{n}-\left(f-f^{T}\right)_{k}\right| \mid \mathcal{F}_{k}\right]
\end{aligned}
$$

and hence

$$
g_{n}^{\sharp}=I(A)\left(f-f^{T}\right)_{n}^{\sharp} .
$$

Then from (2.7), (2.8) and (2.3) for $p=2$ we obtain

$$
\int_{A}\left\{\left(f-f^{T}\right)_{n}^{*}\right\}^{2} d \mathbb{P}=\left\|g_{n}^{*}\right\|_{2} \leq C\left\|g_{n}^{\sharp}\right\|_{2}=C \int_{A}\left\{\left(f-f^{T}\right)_{n}^{\sharp}\right\}^{2} d \mathbb{P} .
$$

Since the above inequality is true for all $A \in \mathcal{F}_{T}$, we obtain (2.6).

THEOREM 2.5. Let $\Phi$ be a non-decreasing continuous function on $[0, \infty)$ with $\Phi(0)=0$ and satisfying the $\triangle_{2}$-condition. If $W \in A_{\infty}(\mathcal{A})$ then there exists a constant $C$ such that

$$
\int_{\Omega} \Phi\left(f^{*}\right) W d \mathbb{P} \leq C \int_{\Omega} \Phi\left(f^{\sharp}\right) W d \mathbb{P}
$$

for all $f \in L^{1}(\Omega, \mathcal{F}, \mathbb{P})$. The constant $C$ depends only on $W, \theta$ and $\Phi$, where $\theta$ is the constant in (2.1).

Proof. Fix $f \in L^{1}$, a stopping time $T$ and an integer $n \geq 0$. Since $g \mapsto g^{*}$ and $g \mapsto g^{\sharp}$ are sublinear, we have

$$
\begin{gathered}
0 \leq f_{n}^{*}-f_{T \wedge n}^{*} \leq\left(f-f^{T}\right)_{n}^{*}, \\
\left(f-f^{T}\right)_{n}^{\sharp} \leq f_{n}^{\sharp}+f_{T \wedge n}^{\sharp} \leq 2 f_{n}^{\sharp} .
\end{gathered}
$$

Therefore by (2.6),

$$
\begin{aligned}
E\left[\left\{f_{n}^{*}-f_{T \wedge n}^{*}\right\}^{2} \mid \mathcal{F}_{T}\right] & \leq E\left[\left\{\left(f-f^{T}\right)_{n}^{*}\right\}^{2} \mid \mathcal{F}_{T}\right] \leq C E\left[\left\{\left(f-f^{T}\right)_{n}^{\sharp}\right\}^{2} \mid \mathcal{F}_{T}\right] \\
& \leq 4 C E\left[\left\{f_{n}^{\sharp}\right\}^{2} \mid \mathcal{F}_{T}\right] .
\end{aligned}
$$


It is easy to see that $f_{T \wedge S}^{*}=\left(f^{S}\right)_{T}^{*}$ and $f_{T \wedge S}^{\sharp}=\left(f^{S}\right)_{T}^{\sharp}$ for all stopping times $T$ and $S$. Then applying Theorem 2.1 we obtain (2.9).

TheOREm 2.6. Let $E$ be a Banach space with the UMD property and with a normalized unconditional basis $\left(e_{j}\right)_{j \geq 1}$, and let $1<p<\infty$. If $f=$ $\sum_{j} f_{j} e_{j} \in L_{E}^{p}$ then $\sum_{j} f_{j}^{*} e_{j}$ and $\sum_{j} f_{j}^{\sharp} e_{j}$ converge in $L_{E}^{p}$ and

$$
\left\|\sum_{j=1}^{\infty} f_{j}^{*} e_{j}\right\|_{L_{E}^{p}} \leq C_{p}\left\|\sum_{j=1}^{\infty} f_{j}^{\sharp} e_{j}\right\|_{L_{E}^{p}},
$$

where $C_{p}$ is a constant depending only on $p, \theta$ and $E$.

Proof. Let $\Phi(t)=t$ and $Z \in A_{\infty}(\mathcal{A})$. Then by Theorem 2.5 there exists a constant $C_{Z}$ such that

$$
\int_{\Omega} f^{*} Z d \mathbb{P} \leq C_{Z} \int_{\Omega} f^{\sharp} Z d \mathbb{P}
$$

for all $f \in L^{1}(\Omega, \mathcal{F}, \mathbb{P})$. Therefore, from Theorem 2.2 there exists a constant $C_{p}$, depending only on $p, \theta$ and $E$, such that (2.12) is true for all $f \in$ $\bigcup_{k=0}^{\infty} L^{p}\left(\Omega, \mathcal{F}_{k}, \mathbb{P} ; E\right)$.

Theorem 2.4 for $\Phi(t)=t^{p}$ and $W=1$ and Theorem 2.3 show that the operator $\tilde{N}\left(\sum_{j} f_{j} e_{j}\right)=\sum_{j} f_{j}^{*} e_{j}$ is well defined and bounded on $L_{E}^{p}$. Since $f_{j}^{\sharp} \leq 2 f_{j}^{*}$, the operator $\widetilde{N}^{\sharp}\left(\sum_{j} f_{j} e_{j}\right)=\sum_{j} f_{j}^{\sharp} e_{j}$ is also well defined and bounded on $L_{E}^{p}$. But $\bigcup_{k=0}^{\infty} L^{p}\left(\Omega, \mathcal{F}_{k}, \mathbb{P} ; E\right)$ is dense in $L_{E}^{p}$ and hence we obtain (2.12) for all $f \in L_{E}^{p}$.

Theorem 2.7. Let $E$ be a Banach space with the UMD property and with a normalized unconditional basis $\left(e_{j}\right)_{j \geq 1}$, let $\Phi$ be a non-decreasing continuous function on $[0, \infty)$ with $\Phi(0)=0$ and satisfying the $\triangle_{2}$-condition, and let $W \in A_{\infty}(\mathcal{A})$. Then there exists a constant $C$, depending only on $W$, $\theta, \Phi$ and $E$, such that for all $f=\sum_{j} f_{j} e_{j} \in \bigcup_{p>1} L_{E}^{p}$,

$$
\int_{\Omega} \Phi\left(\left\|\sum_{j=1}^{\infty} f_{j}^{*} e_{j}\right\|\right) W d \mathbb{P} \leq C \int_{\Omega} \Phi\left(\left\|\sum_{j=1}^{\infty} f_{j}^{\sharp} e_{j}\right\|\right) W d \mathbb{P} .
$$

Proof. We observe that $E$ is a Banach lattice with absolute value $\left|\sum_{j} x_{j} e_{j}\right|$ $=\sum_{j}\left|x_{j}\right| e_{j}$.

Let $1<p<\infty$ and $f=\sum_{j} f_{j} e_{j} \in L_{E}^{p}$. By the proof of Theorem 2.6, $\widetilde{N} f=\sum_{j} f_{j}^{*} e_{j}$ and $\widetilde{N}^{\sharp} f=\sum_{j} f_{j}^{\sharp} e_{j}$ are well defined as functions in $L_{E}^{p}$. We define $U f=\|\tilde{N} f\|, V f=\left\|\tilde{N}^{\sharp} f\right\|$ and $U_{n} f=U\left(E\left[f \mid \mathcal{F}_{n}\right]\right), V_{n} f=$ $V\left(E\left[f \mid \mathcal{F}_{n}\right]\right)$. Since $\left(U_{n} f\right)_{n \geq 0}$ is an increasing sequence and $U_{n} f \rightarrow U f$ in $L^{p}$ as $n \rightarrow \infty$, it follows that $U^{*} f=\sup _{n>0} U_{n} f=U f$. In the same way we show $V^{*} f=V f$. 
If $T$ is a stopping time, we deduce from (2.12) for $p=2$, as in the proof of Lemma 2.1, that there exists a constant $C$, independent of $f, T$ and $n$, such that

$$
E\left[U_{n}^{2}\left(f-f^{T}\right) \mid \mathcal{F}_{T}\right] \leq C E\left[V_{n}^{2}\left(f-f^{T}\right) \mid \mathcal{F}_{T}\right] .
$$

From the inequalities (2.10) and (2.11) we obtain

$$
\left|U_{n} f-U_{T \wedge n} f\right| \leq U_{n}\left(f-f^{T}\right), \quad V_{n}\left(f-f^{T}\right) \leq 2 V_{n} f
$$

and hence

$$
E\left[\left\{U_{n} f-U_{T \wedge n} f\right\}^{2} \mid \mathcal{F}_{T}\right] \leq 4 C E\left[V_{n}^{2} f \mid \mathcal{F}_{T}\right] .
$$

Now, since $\left(f_{j}\right)_{T \wedge S}^{*}=\left(f_{j}^{S}\right)_{T}^{*}$ and $\left(f_{j}\right)_{T \wedge S}^{\sharp}=\left(f_{j}^{S}\right)_{T}^{\sharp}$, it follows that $U_{T \wedge S} f=$ $U_{T} f^{S}$ and $V_{T \wedge S} f=V_{T} f^{S}$ for all stopping times $T$ and $S$. Therefore we can apply Theorem 2.1 to obtain (2.13).

\section{Maximal operators on homogeneous spaces}

LEMMA 3.1 ([23, Lemma 3.21, p. 852]). Let $b$ be a positive integer and let $\lambda=8 \eta^{5}$. Then for each integer $k$ with $-b \leq k \leq b$, there exist an enumerable Borel partition $\mathcal{A}_{k}^{b}$ of $X$ and a positive constant $C$, depending only on $X$, such that:

(i) for all $Q \in \mathcal{A}_{k}^{b}$ with $-b \leq k \leq b$, there exists $x_{Q} \in Q$ such that $B\left(x_{Q}, \lambda^{k}\right) \subset Q \subset B\left(x_{Q}, \lambda^{k+1}\right)$ and $\mu\left(B\left(x_{Q}, \lambda^{k+1}\right)\right) \leq C \mu(Q)$;

(ii) if $-b \leq k<b, Q_{1} \in \mathcal{A}_{k+1}^{b}, Q_{2} \in \mathcal{A}_{k}^{b}$ and $Q_{1} \cap Q_{2} \neq \emptyset$, then $Q_{2} \subset Q_{1}$ and $0<\mu\left(Q_{1}\right) \leq C \mu\left(Q_{2}\right)$.

For a real-valued locally integrable function $f$ on $X$ we define

$$
\begin{aligned}
M_{d}^{b} f(x) & =\sup _{\substack{x \in Q \\
Q \in \mathcal{A}^{b}}} \frac{1}{\mu(Q)} \int_{Q}|f(y)| d \mu(y), \\
M_{d}^{b, \sharp} f(x) & =\sup _{\substack{x \in Q \\
Q \in \mathcal{A}^{b}}} \frac{1}{\mu(Q)} \int_{Q}\left|f(y)-f_{Q}\right| d \mu(y), \\
M^{b} f(x) & =\sup _{B} \frac{1}{\mu(B)} \int_{B}|f(y)| d \mu(y), \\
M^{b, \sharp} f(x) & =\sup _{B} \frac{1}{\mu(B)} \int_{B}\left|f(y)-f_{B}\right| d \mu(y),
\end{aligned}
$$

where the last two suprema are taken over all balls $B=B(a, r)$ such that $x \in B$ and $\lambda^{-b-1} \leq r<\lambda^{b}$, and $\mathcal{A}^{b}=\bigcup_{-b \leq k \leq b} \mathcal{A}_{k}^{b}$.

LEMMA 3.2. Let $E$ be a Banach space with the UMD property and with a normalized unconditional basis $\left(e_{j}\right)_{j \geq 1}$, let $\Phi$ be a non-decreasing continuous function on $[0, \infty)$ with $\Phi(0)=0$ and satisfying the $\triangle_{2}$-condition, and let 
$W \in A_{\infty}\left(\mathcal{A}^{b}\right)$. Then there exists a constant $C$, depending only on $E, \Phi, X$ and $W$, such that for all $f=\sum_{j} f_{j} e_{j} \in L_{E}^{1}$,

$$
\int_{X} \Phi\left(\sup _{k \geq 1}\left\|\sum_{j=1}^{k} M_{d}^{b} f_{j} e_{j}\right\|\right) W d \mu \leq C \int_{X} \Phi\left(M_{d}^{b}(\|f\|)\right) W d \mu .
$$

Proof. Let $\mathcal{A}_{b}^{b}=\left\{Q_{i}^{b}: i \in I_{b}\right\}, I_{b} \subset \mathbb{N}$, and consider the probability measure $\mu_{i}^{b}$ on the Borel subsets of $Q_{i}^{b}$ given by $\mu_{i}^{b}(A)=\mu(A) / \mu\left(Q_{i}^{b}\right)$. Given $f=\sum_{j} f_{j} e_{j} \in L_{E}^{1}$ we have $M_{d}^{b} f_{j}(x)=\left(\left|f_{j}\right|_{\mid Q_{i}^{b}}\right)^{*}(x)$ for $x \in Q_{i}^{b}$, and hence by Lemma 3.1(ii) and Theorem 2.4,

$$
\begin{aligned}
\int_{X} \Phi\left(\sup _{k \geq 1}\right. & \left.\left\|\sum_{j=1}^{k} M_{d}^{b} f_{j}(x) e_{j}\right\|\right) W(x) d \mu(x) \\
& =\sum_{i \in I_{b}} \mu\left(Q_{i}^{b}\right) \int_{Q_{i}^{b}} \Phi\left(\sup _{k \geq 1}\left\|\sum_{j=1}^{k}\left(\left|f_{j}\right|_{\mid Q_{i}^{b}}\right)^{*}(x) e_{j}\right\|\right) W_{\mid Q_{i}^{b}}(x) d \mu_{i}^{b}(x) \\
& \leq C \int_{X} \Phi\left(M_{d}^{b}(\|f\|)(x)\right) W(x) d \mu(x) .
\end{aligned}
$$

LEMMA 3.3. Let $E$ be a Banach space with the UMD property and with a normalized unconditional basis $\left(e_{j}\right)_{j \geq 1}$, let $\Phi$ be a non-decreasing continuous function on $[0, \infty)$ with $\Phi(0)=0$ and satisfying the $\triangle_{2}$-condition, and let $W \in A_{\infty}\left(\mathcal{A}^{b}\right)$. Then there exists a constant $C$, depending only on $E, \Phi, X$ and $W$, such that for all $f=\sum_{j} f_{j} e_{j} \in \bigcup_{p>1} L_{E}^{p}$,

$$
\int_{X} \Phi\left(\left\|\sum_{j=1}^{\infty} M_{d}^{b} f_{j} e_{j}\right\|\right) W d \mu \leq C \int_{X} \Phi\left(\left\|\sum_{j=1}^{\infty} M_{d}^{b, \sharp} f_{j} e_{j}\right\|\right) W d \mu .
$$

Proof. Let $\mu_{i}^{b}, i \in I_{b}$, be as in the proof of Theorem 3.1. Given $f=$ $\sum_{j} f_{j} e_{j} \in \bigcup_{p>1} L_{E}^{p}$ we have $M_{d}^{b} f_{j}(x)=\left(\left|f_{j}\right|_{\mid Q_{i}^{b}}\right)^{*}(x)$ for $x \in Q_{i}^{b}$, and $M_{d}^{b, \sharp}\left(\left|f_{j}\right|\right)(x) \leq 2 M_{d}^{b, \sharp} f_{j}(x)$ for $x \in X$. Therefore by Lemma 3.1(ii) and Theorem 2.7,

$$
\begin{aligned}
\int_{X} \Phi\left(\left\|\sum_{j=1}^{\infty} M_{d}^{b} f_{j}(x) e_{j}\right\|\right) W(x) d \mu(x) \\
\quad=\sum_{i \in I_{b}} \mu\left(Q_{i}^{b}\right) \int_{Q_{i}^{b}} \Phi\left(\left\|\sum_{j=1}^{\infty}\left(\left|f_{j}\right|_{\mid Q_{i}^{b}}\right)^{*}(x) e_{j}\right\|\right) W_{\mid Q_{i}^{b}}(x) d \mu_{i}^{b}(x) \\
\leq C \sum_{i \in I_{b}} \mu\left(Q_{i}^{b}\right) \int_{Q_{i}^{b}} \Phi\left(\left\|\sum_{j=1}^{\infty}\left(\left|f_{j}\right|_{\mid Q_{i}^{b}}\right)^{\sharp}(x) e_{j}\right\|\right) W_{\mid Q_{i}^{b}}(x) d \mu_{i}^{b}(x)
\end{aligned}
$$




$$
\begin{aligned}
& =C \int_{X} \Phi\left(\left\|\sum_{j=1}^{\infty} M_{d}^{b, \sharp}\left(\left|f_{j}\right|\right)(x) e_{j}\right\|\right) W(x) d \mu(x) \\
& \leq C^{\prime} \int_{X} \Phi\left(\left\|\sum_{j=1}^{\infty} M_{d}^{b, \sharp} f_{j}(x) e_{j}\right\|\right) W(x) d \mu(x) .
\end{aligned}
$$

Lemma 3.4. Let $C$ be the constant in Lemma 3.1. Then, for all $1<p$ $\leq \infty$, all real-valued locally integrable functions $f$, and all $x \in X$, we have

$$
\begin{aligned}
A_{p}(X) & \subset A_{p}\left(\mathcal{A}^{b}\right), \\
M_{d}^{b} f(x) & \leq C M^{b} f(x), \\
M_{d}^{b, \sharp} f(x) & \leq 2 C M^{b, \sharp} f(x) .
\end{aligned}
$$

Proof. Let $1<p<\infty, W \in A_{p}(X), Q \in \mathcal{A}_{k}^{b},-b \leq k \leq b$ and $x \in Q$. By Lemma 3.1(i) there exist $x_{Q} \in Q$ and $C>0$ such that $Q \subset B=B\left(x_{Q}, \lambda^{k+1}\right)$ and $\mu(B) \leq C \mu(Q)$. Therefore (1.2) implies that

$$
\left(\frac{1}{\mu(Q)} \int_{Q} W d \mu\right)\left(\frac{1}{\mu(Q)} \int_{Q} W^{-1 /(p-1)} d \mu\right)^{p-1} \leq C^{p} C(p, W) .
$$

Now for a real-valued locally integrable function $f$ we have

$$
\frac{1}{\mu(Q)} \int_{Q}|f(y)| d \mu(y) \leq \frac{C}{\mu(B)} \int_{B}|f(y)| d \mu(y)
$$

and

$$
\begin{aligned}
\frac{1}{\mu(Q)} \int_{Q}\left|f(y)-f_{Q}\right| d \mu(y) & \leq \frac{1}{\mu(Q)} \int_{Q}\left|f(y)-f_{B}\right| d \mu(y)+\left|f_{B}-f_{Q}\right| \\
& \leq \frac{2 C}{\mu(B)} \int_{B}\left|f(y)-f_{B}\right| d \mu(y) \leq 2 C M^{\sharp} f(x) .
\end{aligned}
$$

Thus we obtain (3.3)-(3.5).

Lemma 3.5. Let $b$ be a positive integer. Then there exists a constant $C$, depending only on $X$, such that for all real-valued locally integrable functions $f$ on $X$ and all $x \in B\left(\mathbb{1}, \lambda^{b}\right), \mathbb{1}=\pi(e)$, we have

$$
M^{b} f(x) \leq \frac{C}{\left|\mathcal{G}_{b}\right|} \int_{\mathcal{G}_{b}} M_{d}^{b, g} f(x) d g
$$

where

$$
\mathcal{G}_{b}=\left\{g \in G: d(g \mathbb{1}, \mathbb{1})<\lambda^{b+3}\right\}
$$

and $M_{d}^{b, g} f(x)=R_{g^{-1}} M_{d}^{b} R_{g} f(x), g \in G, x \in X$.

Proof. First we observe that $\left|\mathcal{G}_{b}\right|=\mu\left(B\left(\mathbb{1}, \lambda^{b+3}\right)\right)>0$. Fix $x \in B\left(\mathbb{1}, \lambda^{b}\right)$. From the definition of $M^{b} f(x)$, there exists a ball $B=B(a, r)$ such that 
$x \in B, \lambda^{-b-1} \leq r<\lambda^{b}$ and

$$
M^{b} f(x) \leq \frac{2}{\mu(B)} \int_{B}|f(y)| d \mu(y) .
$$

Let $-b \leq k \leq b$ be such that $\lambda^{k-1} \leq r<\lambda^{k}$. Set

$$
\Omega=\left\{g \in \mathcal{G}_{b} \text { : there exists } Q \in \mathcal{A}_{k+1}^{b} \text { such that } B \subset g^{-1} Q\right\} .
$$

Given $g \in \Omega$, let $Q \in \mathcal{A}_{k+1}^{b}$ be such that $B \subset g^{-1} Q$. By Lemma 3.1(i) there exists $x_{Q} \in Q$ such that $B\left(x_{Q}, \lambda^{k+1}\right) \subset Q \subset B\left(x_{Q}, \lambda^{k+2}\right)$ and hence $g^{-1} Q \subset B\left(g^{-1} x_{Q}, \lambda^{k+2}\right)$. If $s$ is the integer such that $2^{s-1}<\lambda^{3} \leq 2^{s}$, then by the doubling condition we have $\mu\left(B\left(g^{-1} x_{Q}, \lambda^{k+2}\right)\right) \leq A^{s} \mu(B)$ and thus

$$
\frac{1}{\mu(B)} \int_{B}|f(y)| d \mu(y) \leq \frac{A^{s}}{\mu\left(g^{-1} Q\right)} \int_{g^{-1} Q}|f(y)| d \mu(y) .
$$

Therefore from (3.7) we get

$$
M^{b} f(x) \leq 2 A^{s} M_{d}^{b, g} f(x), \quad g \in \Omega .
$$

Now suppose that there exists a positive constant $\alpha$ such that $|\Omega| \geq \alpha\left|\mathcal{G}_{b}\right|$ for all positive integers $b$. Then integrating both sides of the above inequality with respect to the Haar measure $d g$ and on $\Omega$, we get (3.6) for $C=2 A^{s} \alpha^{-1}$.

We will prove that there exists a positive constant $\alpha$, depending only on $X$, such that $|\Omega| \geq \alpha\left|\mathcal{G}_{b}\right|$. Given $y \in X$ we denote by $g_{y}$ an element in $G$ such that $y=g_{y} \mathbb{1}$. Let $z \in g_{x_{Q}} \mathcal{G}_{k-3} g_{x}^{-1}$. Then $z x \in B\left(x_{Q}, \lambda^{k}\right)$ and hence for $y \in B$,

$$
\begin{aligned}
d\left(z y, x_{Q}\right) & \leq \eta\left(d(z y, z x)+d\left(z x, x_{Q}\right)\right) \\
& \leq \eta\left[\eta(d(y, a)+d(a, x))+\lambda^{k}\right] \leq \lambda^{k+1} .
\end{aligned}
$$

Therefore $y \in z^{-1} Q$ and hence

$$
B \subset z^{-1} Q, \quad z \in g_{x_{Q}} \mathcal{G}_{k-3} g_{x}^{-1} .
$$

Set

$$
\Gamma=\left\{Q \in \mathcal{A}_{k+1}^{b}: Q \cap B\left(x, \lambda^{b+2}\right) \neq \emptyset\right\} .
$$

Fix $Q \in \Gamma$ and let $u \in Q \cap B\left(x, \lambda^{b+2}\right), g \in g_{x_{Q}} \mathcal{G}_{k-3}$. Then $g \mathbb{1} \in B\left(x_{Q}, \lambda^{k}\right)$ and

$$
\begin{aligned}
d(g \mathbb{1}, \mathbb{1}) & \leq \eta\left(d\left(g \mathbb{1}, x_{Q}\right)+d\left(x_{Q}, \mathbb{1}\right)\right) \leq \eta\left[\lambda^{k}+\eta\left(d\left(x_{Q}, u\right)+d(u, \mathbb{1})\right)\right] \\
& \leq \eta\left\{\lambda^{k}+\eta\left[\lambda^{k+2}+\eta(d(u, x)+d(x, \mathbb{1}))\right]\right\} \leq 4 \eta^{3} \lambda^{b+2}
\end{aligned}
$$

and hence

$$
d\left(g g_{x}^{-1} \mathbb{1}, \mathbb{1}\right) \leq \eta\left(d\left(g_{x} g^{-1} \mathbb{1}, g_{x} \mathbb{1}\right)+d(x, \mathbb{1})\right) \leq \eta\left(d(g \mathbb{1}, \mathbb{1})+\lambda^{b}\right)<\lambda^{b+3} .
$$

Thus $g \in \mathcal{G}_{b} g_{x}$ and hence

$$
g_{x_{Q}} \mathcal{G}_{k-3} g_{x}^{-1} \subset \mathcal{G}_{b}, \quad Q \in \Gamma
$$


Therefore from (3.8),

$$
\bigcup_{Q \in \Gamma} g_{x_{Q}} \mathcal{G}_{k-3} g_{x}^{-1} \subset \Omega
$$

If $Q, Q^{\prime} \in \mathcal{A}_{k+1}^{b}$ and $Q \neq Q^{\prime}$ then $B\left(x_{Q}, \lambda^{k}\right) \cap B\left(x_{Q^{\prime}}, \lambda^{k}\right)=\emptyset$ and hence

$$
g_{x_{Q}} \mathcal{G}_{k-3} g_{x}^{-1} \cap g_{x_{Q^{\prime}}} \mathcal{G}_{k-3} g_{x}^{-1}=\emptyset .
$$

Then, since $G$ is unimodular (see [16, p. 578]), it follows from (3.9) and the doubling condition that

$$
\begin{aligned}
|\Omega| & \geq\left|\bigcup_{Q \in \Gamma} g_{x_{Q}} \mathcal{G}_{k-3} g_{x}^{-1}\right|=\sum_{Q \in \Gamma}\left|g_{x_{Q}} \mathcal{G}_{k-3}\right| \\
& \geq \sum_{Q \in \Gamma} A^{-s} \mu\left(B\left(x_{Q}, \lambda^{k+2}\right)\right) \\
& \geq A^{-s} \mu\left(\bigcap_{Q \in \Gamma} Q\right) \geq A^{-s} \mu\left(B\left(x, \lambda^{b+2}\right)\right) \geq A^{-2 s}\left|\mathcal{G}_{b}\right| .
\end{aligned}
$$

Proof of Theorem 1.1. Denote by $C$ the greatest of the constants $C$ in (3.1), (3.4) and (3.6), and let $s$ be the integer satisfying $2^{s-1}<C \leq 2^{s}$. Let $f=\sum_{j=1}^{k} f_{j} e_{j} \in L_{E}^{1}$. Since $W \in A_{\infty}(X)$, we can choose $1<p<\infty$ such that $W \in A_{p}(X)$. Then it follows by (1.2) that $R_{g} W \in A_{p}(X)$ and $C\left(p, R_{g} W\right)=C(p, W)$ for all $g \in G$. Therefore by (1.1), (3.1), (3.3), (3.4), (3.6), by Jensen's inequality and Fubini's theorem we have

$$
\begin{aligned}
\int_{B\left(\mathbb{1}, \lambda^{b}\right)} \Phi\left(\left\|\sum_{j=1}^{k} M^{b} f_{j}(x) e_{j}\right\|\right) W(x) d \mu(x) \\
\leq \int_{B\left(\mathbb{1}, \lambda^{b}\right)} \Phi\left(\frac{C}{\left|\mathcal{G}_{b}\right|} \int_{\mathcal{G}_{b}}\left\|\sum_{j=1}^{k} M_{d}^{b, g} f_{j}(x) e_{j}\right\| d g\right) W(x) d \mu(x) \\
\leq \sup _{g \in \mathcal{G}_{b}} c^{s} \int_{X} \Phi\left(\left\|\sum_{j=1}^{k} M_{d}^{b}\left(R_{g} f_{j}\right)(y) e_{j}\right\|\right) R_{g} W(y) d \mu(y) \\
\leq \sup _{g \in \mathcal{G}_{b}} c^{s} C \int_{X} \Phi\left(M_{d}^{b}\left(\left\|R_{g} f\right\|\right)(y)\right) R_{g} W(y) d \mu(y) \\
\leq \sup _{g \in \mathcal{G}_{b}} c^{2 s} C \int_{X} \Phi\left(M^{b}\left(\left\|R_{g} f\right\|\right)(g x)\right) W(x) d \mu(x) \\
\leq c^{2 s} C \int_{X} \Phi(M(\|f\|)(x)) W(x) d \mu(x),
\end{aligned}
$$

since $M\left(\left\|R_{g} f\right\|\right)(g x)=M(\|f\|)(x)$. Now, let $f=\sum_{j=1}^{\infty} f_{j} e_{j}$ and $f^{k}=$ $\sum_{j=1}^{k} f_{j} e_{j}, k \geq 1$. Since the above inequality is true for all $f^{k}, k \geq 1$, 
it follows by the monotone convergence theorem that

$$
\int_{B\left(\mathbb{1}, \lambda^{b}\right)} \Phi\left(\sup _{k \geq 1}\left\|\sum_{j=1}^{k} M^{b} f_{j} e_{j}\right\|\right) W d \mu \leq c^{2 s} C \int_{X} \Phi\left(M^{b}(\|f\|)\right) W d \mu .
$$

Letting $b \rightarrow \infty$ we obtain (1.3).

Finally, let $1<p<\infty, \Phi(t)=t^{p}, W \in A_{p}(X)$ and $f=\sum_{j=1}^{\infty} f_{j} e_{j} \in$ $L_{E}^{p}(W) \cap L_{E}^{1}$. By (1.3) and since the operator $M$ is bounded on $L^{p}(W)$ (see $[6])$,

$$
\left\|\sum_{j=l}^{l+m} M f_{j} e_{j}\right\|_{L_{E}^{p}(W)} \leq C^{1 / p}\left\|M\left(\left\|\sum_{j=l}^{l+m} f_{j} e_{j}\right\|\right)\right\|_{L_{\mathbb{R}}^{p}(W)} \leq C^{\prime}\left\|\sum_{j=l}^{l+m} f_{j} e_{j}\right\|_{L_{E}^{p}(W)} .
$$

From the above inequality we can conclude that $\sum_{j=1}^{\infty} M f_{j} e_{j}$ converges in $L_{E}^{p}(W)$ to a function $\widetilde{M} f$ and

$$
\|\widetilde{M} f\|_{L_{E}^{p}(W)} \leq C^{\prime}\|f\|_{L_{E}^{p}(W)} .
$$

Now let $f=\sum_{j} f_{j} e_{j} \in L_{E}^{p}(W)$ be such that $f_{j} \geq 0$ for all $j \geq 1$. For each $j$, let $\left(f_{j}^{k}\right)_{k \in \mathbb{N}}$ be a sequence of simple functions such that $0 \leq f_{j}^{k} \uparrow f_{j}$ a.e. as $k \rightarrow \infty$. Then $M f_{j}^{k} \uparrow M f_{j}$ and for $f^{k}=\sum_{j} f_{j}^{k} e_{j} \in L_{E}^{p}(W) \cap L_{E}^{1}$ we have $\widetilde{M} f^{k} \uparrow \widetilde{M} f$ a.e. Then

$$
\|\widetilde{M} f\|_{L_{E}^{p}(W)}=\lim _{k \rightarrow \infty}\left\|\widetilde{M} f^{k}\right\|_{L_{E}^{p}(W)} \leq \lim _{k \rightarrow \infty} C^{\prime}\left\|f^{k}\right\|_{L_{E}^{p}(W)}=C^{\prime}\|f\|_{L_{E}^{p}(W)} .
$$

Proof of Theorem 1.2. It follows from Theorem 1.1 that the operator $\widetilde{M}\left(\sum_{j} f_{j} e_{j}\right)=\sum_{j} M f_{j} e_{j}$ is well defined and bounded on $L_{E}^{p}$. Since $M^{\sharp} f_{j} \leq$ $2 M f_{j}$, the operator $\widetilde{M}^{\sharp}\left(\sum_{j} f_{j} e_{j}\right)=\sum_{j} M^{\sharp} f_{j} e_{j}$ is also well defined and bounded on $L_{E}^{p}$.

Denote by $C$ the greatest of the constants $C$ in (3.1), (3.5) and (3.6), and let $s$ be the integer satisfying $2^{s-1}<C \leq 2^{s}$. Since $W \in A_{\infty}(X)$, we can choose $1<p<\infty$ such that $W \in A_{p}(X)$. Then (1.2) implies that $R_{g} W \in A_{p}(X)$ and $C\left(p, R_{g} W\right)=C(p, W)$ for all $g \in G$. Therefore by (1.1), (3.2), (3.3), (3.5), (3.6), by Jensen's inequality and Fubini's theorem we have

$$
\begin{aligned}
\int_{B\left(\mathbb{1}, \lambda^{b}\right)} \Phi\left(\left\|\sum_{j=1}^{\infty} M^{b} f_{j}(x) e_{j}\right\|\right) W(x) d \mu(x) \\
\leq \int_{B\left(\mathbb{1}, \lambda^{b}\right)} \Phi\left(\frac{C}{\left|\mathcal{G}_{b}\right|} \int_{\mathcal{G}_{b}}\left\|\sum_{j=1}^{\infty} M_{d}^{b, g} f_{j}(x) e_{j}\right\| d g\right) W(x) d \mu(x) \\
\leq \sup _{g \in \mathcal{G}_{b}} c^{s} \int_{X} \Phi\left(\left\|\sum_{j=1}^{\infty} M_{d}^{b}\left(R_{g} f_{j}\right)(y) e_{j}\right\|\right) R_{g} W(y) d \mu(y)
\end{aligned}
$$




$$
\begin{aligned}
& \leq \sup _{g \in \mathcal{G}_{b}} c^{s} C \int_{X} \Phi\left(\left\|\sum_{j=1}^{\infty} M_{d}^{b, \sharp}\left(R_{g} f_{j}\right)(y) e_{j}\right\|\right) R_{g} W(y) d \mu(y) \\
& \leq \sup _{g \in \mathcal{G}_{b}} c^{2 s+1} C \int_{X} \Phi\left(\left\|\sum_{j=1}^{\infty} M^{b, \sharp}\left(R_{g} f_{j}\right)(g x) e_{j}\right\|\right) W(x) d \mu(x) \\
& \leq c^{2 s+1} C \int_{X} \Phi\left(\left\|\sum_{j=1}^{\infty} M^{\sharp} f_{j}(x) e_{j}\right\|\right) W(x) d \mu(x),
\end{aligned}
$$

since $M^{\sharp}\left(R_{g} f_{j}\right)(g x)=M^{\sharp} f_{j}(x)$. Letting $b \rightarrow \infty$ we obtain (1.4).

REMARK 3.1. Let $\nu$ be a positive measure on the Borel $\sigma$-field on $X$, finite on compact sets. We define

$$
M \nu(x)=\sup _{B} \frac{1}{\mu(B)} \int_{B} d \nu(y)
$$

where the supremum is taken over all balls $B$ such that $x \in B$. Exactly as in the case of integrable functions, we obtain the following fundamental estimate:

$$
\mu(\{x \in X: M \nu(x)>t\}) \leq \frac{C}{t} \int_{X} d \nu(x) .
$$

It is proved in Macías-Segovia [18] that we can suppose, without loss of generality, that there exist positive constants $C$ and $\alpha, 0<\alpha<1$, such that

$$
|d(x, z)-d(y, z)| \leq C r^{1-\alpha}(d(x, y))^{\alpha}
$$

whenever $d(x, z)$ and $d(y, z)$ are both smaller than $r$. Applying the above inequality we can show that there exists a positive integer $b$ such that for all positive numbers $l, \varepsilon$,

$$
\left\{\begin{array}{l}
B\left(z^{\prime}, \varepsilon\right) \cap(X \backslash B(z, 2 l)) \neq \emptyset \\
B(z, l) \cap B\left(z^{\prime}, \varepsilon\right) \neq \emptyset
\end{array} \Rightarrow B(z, l) \subset B\left(z^{\prime}, 2^{b} \varepsilon\right) .\right.
$$

Fix a real number $\gamma$ with $0<\gamma<1$. We can prove that the function $W(x)=(M \nu(x))^{\gamma}$ is a weight in the Muckenhoupt class $A_{1}(X)$, that is,

$$
\frac{1}{\mu(B)} \int_{B} W(y) d \mu(y) \leq C W(x), \quad x \in B,
$$

for all balls $B$, proceeding exactly as in the Euclidian situation, given e.g. in $\left[13\right.$, Theorem 3.4, Chapter II]. First we fix a ball $B$ and write $\nu=\nu_{1}+$ $\nu_{2}$ where $\nu_{1}(A)=\nu(A \cap B)$. Then we use (3.10) to prove that $W_{1}(x)=$ $\left(M \nu_{1}(x)\right)^{\gamma}$ satisfies $(3.12)$, and use (3.11) to prove that $W_{2}(x)=\left(M \nu_{2}(x)\right)^{\gamma}$ satisfies (3.12). The assertion follows from the inequality $W(x) \leq W_{1}(x)+$ $W_{2}(x)$. 
Now fix a point $p \in X$ and let $\delta_{p}$ denote the Dirac measure concentrated at $p$. Then

$$
W_{p, \gamma}(x)=\left(M \delta_{p}(x)\right)^{\gamma}=\frac{1}{(\mu(B(p, d(p, x))))^{\gamma}}, \quad x \in X,
$$

is a weight in the class $A_{1}(X)$.

Given $W_{1}, W_{2} \in A_{1}(X)$ and $1<p<\infty$, we have $W(x)=W_{1}(x)\left(W_{2}(x)\right)^{1-p}$ $\in A_{p}(X)$ (see e.g. [13]). Therefore given $p_{1}, p_{2} \in X$ and $0<\gamma_{1}, \gamma_{2}<1$, the weight

$$
W(x)=W_{p_{1}, \gamma_{1}}(x) W_{p_{2}, \gamma_{2}}^{1-p}(x)=\frac{\left(\mu\left(B\left(p_{2}, d\left(p_{2}, x\right)\right)\right)\right)^{\gamma_{2}(p-1)}}{\left(\mu\left(B\left(p_{1}, d\left(p_{1}, x\right)\right)\right)\right)^{\gamma_{1}}}
$$

is in the class $A_{p}(X)$.

4. Singular integral operators. In the proof of the following lemma we use the potential-type construction by Bourgain [3].

LEMMA 4.1. Let $E$ be a Banach space with the UMD property and with a normalized unconditional basis $\left(e_{j}\right)_{j \geq 1}$, let $1<p<\infty$, and let $W \in A_{p}(X)$. Then there exist positive constants $C_{p}$ and $r>1$, depending only on $p, W$, $X$ and $E$, such that

$$
\left\|\sum_{j=1}^{\infty} M_{r} f_{j} e_{j}\right\|_{L_{E}^{p}(W)} \leq C_{p}\left\|\sum_{j=1}^{\infty} f_{j} e_{j}\right\|_{L_{E}^{p}(W)}
$$

for all $f=\sum_{j} f_{j} e_{j} \in L_{E}^{p}(W)$, where $M_{r} g=\left(M\left(|g|^{r}\right)\right)^{1 / r}$.

Proof. Let $\Phi(t)=t^{p}$, let $C$ be the constant in (1.3), and let $g=$ $\sum_{j} g_{j} e_{j} \in L_{E}^{p}(W)$. For each $j \geq 1$ we define

$$
\psi_{j}=\sum_{i=0}^{\infty}\left(2 C^{1 / p}\right)^{-i} M^{(i)} g_{j},
$$

where $M^{(i)} g_{j}$ is defined inductively by $M^{(0)} g_{j}=\left|g_{j}\right|, M^{(i+1)} g_{j}=M\left(M^{(i)} g_{j}\right)$. We have

$$
M \psi_{j} \leq 2 C^{1 / p} \psi_{j}
$$

and hence the weights $\psi_{j}, j \geq 1$, are by definition uniformly in the class $A_{1}(X)$. It follows from the reverse Hölder inequality (see Calderón [6]) that there exist positive constants $C^{\prime}$ and $r, r>1$, depending only on $p$ and $C$, such that

$$
\left(\frac{1}{\mu(B)} \int_{B} \psi_{j}^{r} d \mu\right)^{1 / r} \leq \frac{C^{\prime}}{\mu(B)} \int_{B} \psi_{j} d \mu
$$

for all balls $B$ and all $j \geq 1$. Therefore

$$
M_{r} g_{j}(x) \leq M_{r} \psi_{j}(x) \leq C^{\prime} M \psi_{j}(x) \leq 2 C^{1 / p} C^{\prime} \psi_{j}(x) .
$$


But by Theorem 1.1,

$$
\left\|\sum_{j=1}^{\infty} \psi_{j} e_{j}\right\|_{L_{E}^{p}(W)} \leq \sum_{i=0}^{\infty}\left(2 C^{1 / p}\right)^{-i}\left\|\sum_{j=1}^{\infty} M^{(i)} g_{j} e_{j}\right\|_{L_{E}^{p}(W)} \leq 2\|g\|_{L_{E}^{p}(W)}
$$

and hence

$$
\left\|\sum_{j=1}^{\infty} M_{r} g_{j} e_{j}\right\|_{L_{E}^{p}(W)} \leq 2 C^{1 / p} C^{\prime}\left\|\sum_{j=1}^{\infty} \psi_{j} e_{j}\right\|_{L_{E}^{p}(W)} \leq 4 C^{1 / p} C^{\prime}\|g\|_{L_{E}^{p}(W)} .
$$

LEMMA 4.2. Let $T$ be a singular integral operator bounded on $L^{r}(X)$ for some $r, 1<r<\infty$. Assume that the kernel $K$ of $T$ satisfies $\left(H_{\infty}^{\prime}\right)$ and $K(g x, g y)=K(x, y)$ for all $x, y \in X$ and $g \in G$. Then there exists $a$ constant $C_{r}$ such that

$$
M^{\sharp}(T f)(x) \leq C_{r} M_{r} f(x), \quad f \in L_{\mathrm{c}}^{\infty}(X) .
$$

Proof. Fix $x_{0} \in X, l>0$ and let $B=B\left(x_{0}, l\right), B^{2}=B\left(x_{0}, 2 l\right)$. For $f \in L_{c}^{\infty}(X)$ we set $g=f \chi_{B^{2}}, h=f-g$. Since $T$ is bounded on $L^{r}(X)$, for all $z \in B$ we have

$$
\begin{aligned}
\frac{1}{\mu(B)} \int_{B}\left|T g(x)-(T g)_{B}\right| d \mu(x) & \leq \frac{2}{\mu(B)} \int_{B}|T g(x)| d \mu(x) \\
& \leq C_{r}\left(\frac{1}{\mu(B)} \int_{B^{2}}|g(x)|^{r} d \mu(x)\right)^{1 / r} \\
& \leq C_{r} A^{1 / r} M_{r} g(z)
\end{aligned}
$$

Now let $x \in B$ and $g \in G$ be such that $g x_{0}=\mathbb{1}, \bar{x}=g x$ and

$$
S_{j}(\bar{x})=\left\{t: 2^{j} d(\bar{x}, \mathbb{1})<d(t, \mathbb{1}) \leq 2^{j+1} d(\bar{x}, \mathbb{1})\right\} .
$$

Then by the $\left(H_{\infty}^{\prime}\right)$ condition, for all $z \in B$,

$$
\begin{aligned}
\mid T h(x)- & T h\left(x_{0}\right)\left|\leq \int_{X \backslash B^{2}}\right| K(x, y)-K\left(x_{0}, y\right)|| h(y) \mid d \mu(y) \\
& \leq \int_{d(t, \mathbb{1})>2 d(\bar{x}, \mathbb{1})}\left|K^{\prime}(t, \bar{x})-K^{\prime}(t, \mathbb{1})\right|\left|R_{g} h(t)\right| d \mu(t) \\
& \leq C \sum_{j=1}^{\infty} \int_{S_{j}(\bar{x})} \frac{d(\bar{x}, \mathbb{1})}{d(t, \mathbb{1}) \mu(B(\mathbb{1}, d(t, \mathbb{1})))}\left|R_{g} h(t)\right| d \mu(t) \\
& \leq A C \sum_{j=1}^{\infty} \frac{2^{-j}}{\mu\left(B\left(x_{0}, 2^{j+1} d\left(x, x_{0}\right)\right)\right)} \int_{g B\left(x_{0}, 2^{j+1} d\left(x, x_{0}\right)\right)}\left|R_{g} h(t)\right| d \mu(t)
\end{aligned}
$$




$$
\begin{aligned}
& =A C \sum_{\substack{1 \leq j<\infty \\
2^{j} d\left(x, x_{0}\right)>l}}^{\infty} \frac{2^{-j}}{\mu\left(B\left(x_{0}, 2^{j+1} d\left(x, x_{0}\right)\right)\right)} \int_{B\left(x_{0}, 2^{j+1} d\left(x, x_{0}\right)\right)}|h(y)| d \mu(y) \\
& \leq A C M_{r} h(z)
\end{aligned}
$$

and hence

$$
\begin{aligned}
\frac{1}{\mu(B)} \int_{B}\left|T h(x)-(T h)_{B}\right| d \mu(x) & \leq \frac{2}{\mu(B)} \int_{B}\left|T h(x)-T h\left(x_{0}\right)\right| d \mu(x) \\
& \leq 2 A C M_{r} h(z) .
\end{aligned}
$$

Thus for all $z \in X$,

$$
\begin{aligned}
& M^{\sharp}(T f)(z) \\
& \leq \sup _{B \ni z} \frac{1}{\mu(B)} \int_{B}\left|T g(x)-(T g)_{B}\right| d \mu(x)+\sup _{B \ni z} \frac{1}{\mu(B)} \int_{B}\left|T h(x)-(T h)_{B}\right| d \mu(x) \\
& \leq C_{r}^{\prime} M_{r} f(z) .
\end{aligned}
$$

Proof of Theorem 1.3. Fix $1<p<\infty$ and $W \in A_{p}(X)$, and let $r$ and $C_{p}$ be the constants in Lemma 4.1. Then (1.4) for $\Phi(t)=t^{p}$ shows, by (1.5) and (4.1), that for all $f=\sum_{j} f_{j} e_{j}$ with $f_{j} \in L_{\mathrm{c}}^{\infty}(X)$ for $j \geq 1$, and all positive integers $l$ and $m$,

$$
\begin{aligned}
\left\|\sum_{j=l}^{l+m} T_{j} f_{j} e_{j}\right\|_{L_{E}^{p}(W)} & \leq\left\|\sum_{j=l}^{l+m} M\left(T_{j} f_{j}\right) e_{j}\right\|_{L_{E}^{p}(W)} \\
& \leq C^{1 / p}\left\|\sum_{j=l}^{l+m} M^{\sharp}\left(T_{j} f_{j}\right) e_{j}\right\|_{L_{E}^{p}(W)} \\
& \leq C^{1 / p} C_{r}\left\|\sum_{j=l}^{l+m} M_{r} f_{j} e_{j}\right\|_{L_{E}^{p}(W)} \\
& \leq C^{1 / p} C_{r} C_{p}\left\|\sum_{j=l}^{l+m} f_{j} e_{j}\right\|_{L_{E}^{p}(W)}
\end{aligned}
$$

The above inequality implies that the sequence of partial sums of the series $\sum_{j} T_{j} f_{j} e_{j}$ is a Cauchy sequence in $L_{E}^{p}(W)$ and hence it converges in $L_{E}^{p}(W)$. Putting $l=1$ and letting $m \rightarrow \infty$ we obtain (1.6).

Proof of Corollary 1.1. For all $0 \leq r \leq 1$ and all $x, y \in S^{n}$,

$$
\begin{array}{r}
|y-(y \cdot x) x| \leq 2|y-x|, \\
|y-\mathbb{1}-[(y-\mathbb{1}) \cdot x] x| \leq 2|y-\mathbb{1}|,
\end{array}
$$




$$
\begin{aligned}
|(x \cdot \mathbb{1}) \mathbb{1}-(x \cdot y) y| & \leq 2|y-\mathbb{1}|, \\
|y-x| & \leq 2|y-r x|,
\end{aligned}
$$

and for all $0 \leq r \leq 1$ and all $x, y \in S^{n}$ such that $|x-\mathbb{1}|>2|y-\mathbb{1}|$,

$$
\begin{aligned}
& \frac{1}{2}|\mathbb{1}-r x|<|y-r x|<2|\mathbb{1}-r x|, \\
& \frac{1}{2}|x-r \mathbb{1}|<|x-r y|<2|x-r \mathbb{1}| .
\end{aligned}
$$

Now fix $0 \leq r \leq 1$ and $x, y \in S^{n}$ such that $|x-\mathbb{1}|>2|y-\mathbb{1}|$. Then by (4.6),

$$
\begin{aligned}
|| \mathbb{1}-\left.r x\right|^{n+1}-\mid y & -\left.r x\right|^{n+1} \mid \\
& \leq\left(|\mathbb{1}-r x|^{n}+|\mathbb{1}-r x|^{n-1}|y-r x|+\ldots+|y-r x|^{n}\right) \\
& \leq C|y-\mathbb{1}||\mathbb{1}-r x|^{n}
\end{aligned}
$$

and hence by (4.2), (4.3), (4.5) and (4.6) we obtain

$$
\begin{aligned}
\left|s_{r}(x, y)-s_{r}(x, \mathbb{1})\right| \leq & \frac{2}{\omega_{n}}|y-(y \cdot x) x| \frac{|| \mathbb{1}-\left.r x\right|^{n+1}-|y-r x|^{n+1} \mid}{|y-r x|^{n+1}|\mathbb{1}-r x|^{n+1}} \\
& +\frac{2}{\omega_{n}} \frac{|y-\mathbb{1}-[(y-\mathbb{1}) \cdot x] x|}{|\mathbb{1}-r x|^{n+1}} \\
\leq & C_{1} \frac{|y-\mathbb{1}|}{|x-\mathbb{1}|^{n+1}}, \\
\left|t_{r}(x, y)-t_{r}(x, \mathbb{1})\right| \leq & \frac{n-2}{2 r} \int_{0}^{r}\left|s_{\varrho}(x, y)-s_{\varrho}(x, \mathbb{1})\right| d \varrho \\
= & \frac{(n-2) C_{1}}{2} \frac{|y-\mathbb{1}|}{|x-\mathbb{1}|^{n+1}}, \\
\left|K_{i, j}^{r}(x, y)-K_{i, j}^{r}(x, \mathbb{1})\right| \leq & \left|x_{i} y_{j}-x_{j} y_{i}\right| \frac{|| \mathbb{1}-\left.r x\right|^{n+1}-|y-r x|^{n+1} \mid}{|y-r x|^{n+1}|\mathbb{1}-r x|^{n+1}} \\
& +\left|x_{i}\left(y_{j}-\mathbb{1}_{j}\right)-x_{j}\left(y_{i}-\mathbb{1}_{i}\right)\right| \frac{1}{|\mathbb{1}-r x|^{n+1}} \\
\leq & C_{2} \frac{|y-\mathbb{1}|}{|x-\mathbb{1}|^{n+1}} .
\end{aligned}
$$

Since $|\mathbb{1}-r x| \geq 1-r$,

$$
\begin{aligned}
\left|P_{r}(x, y)-P_{r}(x, \mathbb{1})\right| & \leq \frac{1-r^{2}}{\omega_{n}} \frac{|| \mathbb{1}-\left.r x\right|^{n+1}-|y-r x|^{n+1} \mid}{|y-r x|^{n+1}|\mathbb{1}-r x|^{n+1}} \\
& \leq C_{3} \frac{|y-\mathbb{1}|}{|x-\mathbb{1}|^{n+1}}
\end{aligned}
$$

and hence

$$
|K(x, y)-K(x, \mathbb{1})| \leq C_{3} \frac{|y-\mathbb{1}|}{|x-\mathbb{1}|^{n+1}}
$$


Therefore the kernels $s_{r}, t_{r}, K_{i, j}^{r}$ and $K$ satisfy condition $\left(H_{\infty}\right)$ uniformly for all $0 \leq r \leq 1, i, j \in\{1, \ldots, n+1\}$. In the same way we can use (4.2), $(4.4),(4.5)$ and (4.7) to show that $s_{r}, t_{r}, K_{i, j}^{r}$ and $K$ satisfy $\left(H_{\infty}^{\prime}\right)$ uniformly for all $r, i, j$. The conclusion of Corollary 1.1 follows from the remark given above of the statement of this corollary and from Theorem 1.4.

\section{References}

[1] A. Benedek, A. Calderón and R. Panzone, Convolution operators on Banach space valued functions, Proc. Natl. Acad. Sci. USA 48 (1962), 356-365.

[2] B. Bordin and D. L. Fernandez, Singular integral operators with operator-valued kernels on spaces of homogeneous type, Z. Anal. Anwendungen 11 (1992), 153-166.

[3] J. Bourgain, Extension of a result of Benedek, Calderón and Panzone, Ark. Mat. 22 (1984), 91-95.

[4] -, Vector-valued singular integrals and the $H^{1}-B M O$ duality, in: Probability Theory and Harmonic Analysis (Cleveland, OH, 1983), Monogr. Textbooks Pure Appl. Math. 98, Dekker, New York, 1986, 1-19.

[5] D. L. Burkholder, A geometric condition that implies the existence of certain singular integrals of Banach-space-valued functions, in: Proc. Conf. Harmonic Analysis in Honor of Antoni Zygmund, Wadsworth, Belmont, CA, 1983, 270-286.

[6] A. P. Calderón, Inequalities for the maximal function relative to a metric, Studia Math. 57 (1976), 297-306.

[7] R. R. Coifman et G. Weiss, Analyse Harmonique Non-Commutative sur Certains Espaces Homogènes, Lecture Notes in Math. 242, Springer, Berlin, 1971.

[8] C. Fefferman and E. M. Stein, Some maximal inequalities, Amer. J. Math. 93 (1971), $107-115$.

[9] - - - $H^{p}$ spaces of several variables, Acta Math. 129 (1972), 137-193.

[10] A. M. Garcia, Martingale Inequalities: Seminar Notes on Recent Progress, Benjamin, 1973.

[11] J. García-Cuerva, R. A. Macías and J. L. Torrea, The Hardy-Littlewood property of Banach lattices, Israel J. Math. 83 (1993), 177-201.

[12] - - - - Maximal operators and B.M.O. for Banach lattices, Proc. Edinburgh Math. Soc. 41 (1998), 585-609.

[13] J. García-Cuerva and J. L. Rubio de Francia, Weighted Norm Inequalities and Related Topics, North-Holland Math. Stud. 116, North-Holland, 1985.

[14] E. Harboure, R. A. Macías, C. Segovia and J. L. Torrea, Some estimates for maximal functions on Köthe function spaces, Israel J. Math. 90 (1995), 349-371.

[15] M. Izumisawa, Weighted norm inequality for operator on martingales, Tôhoku Math. J. 32 (1980), 1-8.

[16] A. Korányi and S. Vági, Singular integrals on homogeneous spaces and some problems of classical analysis, Ann. Scuola Norm. Sup. Pisa 25 (1971), 575-648.

[17] D. A. Levine, Systems of singular integrals operators on spheres, Trans. Amer. Math. Soc. 144 (1969), 493-522.

[18] R. Macías and C. Segovia, Lipschitz functions on spaces of homogeneous type, Adv. in Math. 33 (1979), 257-270.

[19] K. Phillips and M. H. Taibleson, Singular integrals in several variables over a local field, Pacific J. Math. 30 (1969), 209-231. 
[20] J. L. Rubio de Francia, Martingale and integral transforms of Banach space valued functions, in: Probability and Banach Spaces, Lecture Notes in Math. 1221, Springer, Berlin, 1985, 195-222.

[21] J. L. Rubio de Francia, F. Ruiz and J. L. Torrea, Calderón-Zygmund theory for operator-valued kernels, Adv. Math. 62 (1986), 7-48.

[22] F. J. Ruiz and J. L. Torrea, Vector-valued Calderón-Zygmund theory and Carleson measures on spaces of homogeneous nature, Studia Math. 88 (1988), 221-243.

[23] E. Sawyer and R. L. Wheeden, Weighted inequalities for fractional integrals on euclidean and homogeneous spaces, Amer. J. Math. 114 (1992), 813-874.

[24] M. H. Taibleson, The existence of natural field structures for finite dimensional vector spaces over local fields, Pacific J. Math. 63 (1976), 545-551.

[25] S. A. Tozoni, Weighted inequalities for vector operators on martingales, J. Math. Anal. Appl. 191 (1995), 229-249.

[26] - Vector-valued extentions of operators on martingales, ibid. 201 (1996), 128-151.

Instituto de Matemática

Universidade Estadual de Campinas

Caixa Postal 6065

13.081-970 Campinas - SP, Brazil

E-mail: tozoni@ime.unicamp.br

Received August 14, 2002

Revised version June 16, 2003 